\title{
Beyond horizontal gene transfer: the role of plasmids in bacterial evolution
}

Jeronimo Rodriguez-Beltran ${ }^{1 \dagger}$, Javier DelaFuente ${ }^{1}$, Ricardo Leon-Sampedro ${ }^{1}, \mathrm{R}$. Craig MacLean ${ }^{2}$ and Alvaro San Millan ${ }^{1,3+}$

${ }^{1}$ Department of Microbiology, Hospital Universitario Ramon y Cajal, Instituto Ramón y Cajal de Investigación Sanitaria (IRYCIS), Madrid, Spain.

${ }^{2}$ Department of Zoology, University of Oxford, Oxford, UK.

${ }^{3}$ Centro Nacional de Biotecnología-CSIC, Madrid, Spain.

†e-mail: jeronimo.rodriguez.beltran@gmail.com; asanmillan@cnb.csic.es

\section{Abstract}

Plasmids have a key role in bacterial ecology and evolution because they mobilize accessory genes by horizontal gene transfer. However, recent studies have revealed that the evolutionary impact of plasmids goes above and beyond being mere gene delivery platforms. Plasmids are usually kept at multiple copies per cell, producing islands of polyploidy in the bacterial genome. As a consequence, the evolution of plasmid-encoded genes is governed by a different set of rules than those affecting chromosomal genes, and these rules are shaped by unusual concepts in bacterial genetics such as genetic dominance, heteroplasmy or segregational drift. In this Review, we discuss recent advances that underscore the importance of plasmids in bacterial ecology and evolution beyond horizontal gene transfer. We focus on new evidence that suggests that plasmids might accelerate bacterial evolution, mainly by promoting the evolution of plasmid-encoded genes, but also by enhancing the adaptation of their host chromosome. Finally, we integrate the most relevant 
theoretical and empirical studies providing a global understanding of the forces that govern plasmid-mediated evolution in bacteria.

\section{[H1] Introduction}

Ever since small genetic molecules competed for self-replication in the early origins of life, the evolution of every organism has been shaped by the coexistence of diverse DNA molecules within the cell. Among these, plasmids - autonomouslyreplicating DNA molecules that stably coexist with chromosomes — stand out as drivers of horizontal gene transfer [G] (HGT) in prokaryotic evolution. The word plasmid was first coined by Joshua Lederberg in 1952 as a "generic term for any extra-chromosomal hereditary determinant"1, but it was not until the characterization of the first conjugative plasmids in the early 1960 s that the term rose in popularity ${ }^{2}$. Since then, the study of plasmid biology has produced a remarkable wealth of knowledge, which has nourished the fields of bacterial genetics and molecular biology, among others.

From an evolutionary perspective, plasmids may be considered selfish (or parasitic) genetic elements, as they do not necessarily share evolutionary interests with the chromosome ${ }^{3,4}$. Plasmids encode genes to ensure their maintenance and transmission that are often disadvantageous to the host, such as post-segregational killing systems $[\mathbf{G}]^{5}$. However, plasmids have an important role in bacterial evolution by transferring beneficial traits within and between species of bacteria, positively contributing to host fitness. Therefore, there is a clear trade-off between plasmid parasitic and mutualistic lifestyles, challenging our understanding of their existence conditions (Box 1). 
Recent work has shown that plasmid-carried genes evolve in fundamentally different ways from chromosomal genes, suggesting that plasmids are more than just vehicles for gene transfer. Deciphering the evolutionary opportunities and constraints associated with plasmids is key to understanding and predicting the evolution of plasmid traits, such as antibiotic resistance and bacterial virulence. In this Review, we discuss recent advances that highlight plasmids as catalysts of bacterial evolution beyond HGT, with a special focus on the evolution of antibiotic resistance.

\section{[H1] Anatomy of plasmids}

\section{[H2] Plasmid prevalence and types}

Plasmids are remarkably diverse. Plasmids vary in size, copy number, GC content, replication mechanism, transmission mode, DNA topology (that is, circular or linear), genetic cargo and host range, among other features. From the small parasitic $2 \mu \mathrm{m}$ plasmid of Saccharomyces cerevisiae to the worrisome enterobacterial antibiotic resistance plasmids, the wide variety of plasmid types mirrors the extreme diversity of their hosts, which span all the domains of life $^{6}$. Bacterial plasmids have received particular attention because of their prevalence (FIG. 1a) and are the main subject of this Review. In spite of their diversity, bacterial plasmids can largely be classified into two groups (FIG. 1b): low-copy number plasmids (LCPs) and high-copy number plasmids (HCPs). LCPs are typically large (from tens to hundreds of kilobases), they present a low copy number, and are frequently conjugative. On the other hand, HCPs are in general small, kept at high copy numbers and typically lack a functional conjugative system, but some of them can be mobilised through the conjugative apparatus of other coexisting plasmids ${ }^{7}$. Plasmids can be further classified based on replication $^{8 \square}$ and mobility proteins ${ }^{9}$, or based on general similarity in genetic content and nucleotide homology ${ }^{10-12}$. However, for the purposes of this Review, we focus on the broad scale distinction between LCPs and HCPs. 


\section{[H2] Plasmid-encoded traits}

Plasmids encode a vast catalogue of traits that promote their own survival and increase the range of environmental conditions in which their host can thrive. Plasmids carry a backbone or 'core' genes that are instrumental to their vertical and horizontal transmission such as those coding replicative and conjugative proteins. Additionally, plasmids can encode specialized systems to improve their transmission and stability. Some examples include anti-restriction proteins that contribute to the evasion of host defences ${ }^{13,14}$, post-segregational killing systems that ensure plasmid persistence $^{5}$, and type IV CRISPR-Cas systems that mediate inter-plasmid competition ${ }^{15}$.

Although some plasmids are cryptic and do not encode any known function beyond those devoted to their own survival ${ }^{16,17}$, most plasmids encode 'accessory' genes that can expand the ecological niche of their hosts by degrading toxic compounds or providing new metabolic capabilities. For instance, plasmids carrying virulence and antibiotic resistance genes are key contributors to the uncontrollable spread of bacterial pathogens, particularly in clinical settings ${ }^{18,19}$. Beyond human health, plasmid-associated traits are responsible for major transitions in the evolution of several bacterial clades. Some prominent examples include the ability to undergo anoxygenic photosynthesis in marine bacteria of the Rhodobacteriaceae family ${ }^{20}$, and the metabolic plasmids of the aphid endosymbiont Buchnera aphidicola, which are responsible for synthesizing essential amino acids and vitamins required for the bacterium-aphid symbiotic relationship, and have had a key role in the transition of B. aphidicola from free-living to obligate endosymbiont ${ }^{21}$. These examples, and several others ${ }^{22,23}$, highlight the crucial importance of plasmid traits for the diversity of bacterial lifestyles. 
[H2] Plasmids as vectors of horizontal gene transfer

HGT is a major force fuelling prokaryotic evolution ${ }^{24}$. HGT is pervasive and allows taxonomically different organisms to share a common genetic pool which blurs the boundaries between distinct phylogenetic clades and explains why closely related prokaryotes greatly differ in gene content ${ }^{25}$. Plasmids are key drivers of HGT and can be transferred at seemingly high rates through several mechanisms, mainly conjugation (including plasmid mobilization and conduction), but also transduction, transformation and vesiduction (Box 2). Several factors are known to affect the transferability of plasmids in natural settings ${ }^{26,27}$, yet direct measurements of transfer in the environment are still scarce (reviewed in REF. ${ }^{28}$ ). Recent studies have focused on quantifying the extent of plasmid-mediated gene transfer in the human gut. Longitudinal whole genome sequencing of bacterial samples from human donors demonstrated that plasmid transfer is pervasive, even in the absence of antibiotic treament ${ }^{29-31}$. This view has been further confirmed using tailored CRISPR spacer acquisition systems to record the history and diversity of HGT among human microbiota ${ }^{32}$. Although plasmid transfer is one of the major drivers of prokaryotic evolution, this Review focuses on the less explored mechanisms of plasmidmediated evolution that go beyond HGT, so we direct the reader to excellent Reviews on plasmids and HGT for further information ${ }^{24,28}$.

\section{[H2] Multi-copy nature of plasmids}

Arguably, one of the most far reaching properties of plasmids is that they are kept at several copies per cell. Plasmid copy number (PCN) ranges from only slightly higher than chromosomal copy number in LCPs, to tens of copies per cell in $\mathrm{HCPs}^{33}$. This fundamental difference in PCN is associated with the presence or absence of active partitioning systems. HCPs generally lack these systems, and therefore they rely on a high PCN to be stably inherited, avoiding loss during plasmid segregation [G] at 
cell division. Despite these differences, all plasmids (LCPs and HCPs) present on average more than one copy per bacterial cell.

Plasmids dynamically adjust their copy number during bacterial growth through negative feed-back loops ${ }^{34}$. These systems ensure that mean copy number is constant at the population level, yet there is substantial stochastic heterogeneity at the cellular level ${ }^{35-37}$, with cells showing up to a 10 -fold higher copy number than others $^{38}$. Additionally, although $\mathrm{PCN}$ is an evolutionary robust trait ${ }^{39}$, certain mutations in replicative genes can stably increase copy number and are routinely isolated in laboratory and clinical settings ${ }^{39-43}$. This variability in plasmid copy number can be further compounded in cases where environmental cues modulate $\mathrm{PCN}^{44-48}$. This is the case for the virulence plasmids of two phylogenetically distant bacterial species: Yersinia spp. ${ }^{45}$ and Agrobacterium tumefaciens ${ }^{44,48}$. These pathogens carry plasmids that are essential for their infectious lifestyle, the copy number of which is up-regulated in response to signals emitted by their target cells ${ }^{44,45}$. The key insight from these examples is that $\mathrm{PCN}$ is a plastic trait, and $\mathrm{PCN}$ varies widely between cells, even if they belong to the same clonal population.

It is becoming clear that most bacterial chromosomes are effectively polyploid at least in some growth conditions ${ }^{49}$. For instance, increased ploidy levels are typically associated with fast growth in Escherichia coli, but not with slow growth or stationary phase $^{49}$. By contrast, plasmids are stable polyploid molecules that remain multi-copy throughout the whole bacterial life cycle ${ }^{50,51}$. In the following sections we focus on the evolutionary consequences of the multi-copy nature of plasmids, although some of the principles addressed below could also be extrapolated to the chromosomes of polyploid bacteria. 


\section{[H1] Plasmid copy number and evolution}

\section{[H2] Increased gene expression}

Owing to their copy number, plasmid genes usually have higher gene dosage effects [G] than chromosomal genes and, as a result, plasmid genes tend to be highly expressed ${ }^{52}$. Consequently, plasmids have traditionally been harnessed for largescale protein production. From an evolutionary perspective, high gene expression is a major barrier to $\mathrm{HGT}^{53}$, yet it can be beneficial under several circumstances. For instance, some members of the genus Aureimonas lack a chromosomal copy of the rRNA ( $r r n)$ operon. Instead, these bacteria carry their sole $r r n$ operon on a small HCP held at 18 to 34 copies per cell 22 . High gene dosage of $r r n$ operons is known to promote rapid adaptation to changing environmental conditions by facilitating a sudden increase in rRNA expression ${ }^{54}$. Thus, the Aureimonas spp. plasmid-encoded rrn might help to cope with the common fluctuating conditions that these bacteria face in their natural habitat 22,55 . Other paradigmatic examples are provided by antimicrobial resistance enzymes that are typically plasmid-encoded. For instance, $\beta$ lactamases - enzymes that are able to degrade $\beta$-lactam antibiotics - are found both on plasmids and chromosomes, but resistance levels are higher (up to 128-fold) when these enzymes are plasmid-encoded ${ }^{40,42,56}$. Therefore, plasmid-driven gene dosage effects might help to predict which antibiotic resistance genes are more likely to associate with plasmids (that is, those that provide more resistance with increased gene dosage).

As stated in the previous section, subpopulations of cells with a relatively high PCN randomly appear as a by-product of plasmid segregation and replication dynamics. Thus, in any plasmid-carrying bacterial population of reasonable size, subpopulations 
showing elevated PCN are likely to exist. This $\mathrm{PCN}$ intrapopulation variability might lead to potentially beneficial phenotypic plasticity, by which only a fraction of cells show increased expression of plasmid-encoded genes. An illustrative example is provided by bacterial heteroresistance, a phenomenon by which clonal subpopulations of bacteria show lower susceptibility to an antibiotic insult than the main population ${ }^{57}$. Several genetic mechanisms underlie heteroresistance (reviewed in $R E F .{ }^{58}$ ), although alteration of gene dosage is the most common among Gramnegative bacteria ${ }^{59}$. By amplifying gene dosage of genes that confer low level resistance, heteroresistant bacteria can acquire transient high-level antimicrobial resistance ${ }^{59}$. A recent study found that tandem genetic duplications [G] were involved in most of heteroresistance instances ${ }^{59}$. Crucially, when antibiotic resistance genes were plasmid-encoded, the effect of tandem duplications was further enhanced by increases in $\mathrm{PCN}^{59}$, suggesting that both mechanisms act synergistically to drastically increase gene dosage in a fraction of the bacterial population. These resistant subpopulations might lead to antibiotic treatment failure, as they are likely to be undetected by conventional antimicrobial susceptibility testing ${ }^{58}$.

Colicin production offers another paradigmatic example of the effects of PCN in microbial lifestyles. Colicins are plasmid-encoded toxins produced by enteric bacteria that shape microbial interactions by killing competing bacteria ${ }^{60}$. Colicin production leads to producer cell death as a side-effect of colicin release, and thus it is extremely costly to the population ${ }^{61}$. As a consequence, colicin production is stochastically triggered only in a small fraction of cells ${ }^{61,62}$. Among the genetic factors that control colicin synthesis, PCN variability has a crucial role, as cells with high $\mathrm{PCN}$ are more likely to undergo colicin release ${ }^{62}$.

These studies suggest that population heterogeneity in PCN might work as a bethedging [G] strategy: a fraction of the population is best suited to survive an 
environmental perturbation, but do so at the cost of overexpressing plasmid-encoded genes, which produces a competitive disadvantage when selection for high expression is absent ${ }^{63}$.

\section{[H2] Increased genetic variability}

Mutation and recombination [G] provide the evolutionary 'raw material' in which selection acts in classic models of evolution. Mutation rates per gene linearly increase with gene copy number, and recombination is more frequent in plasmids than chromosomes ${ }^{64,65}$, suggesting that plasmid-encoded genes should evolve faster than chromosomal genes. This is indeed the case in the evolution of $\beta$-lactamases. Certain mutations in the bla $\mathrm{TEM}_{\mathrm{T}-1}$ gene expand its range of activity, conferring resistance to a wide variety of $\beta$-lactam antibiotics. One study evolved wild-type and mutator populations of $E$. coli carrying bla TEM -1 to increasing concentrations of cefotaxime, a third-generation cephalosporin. Interestingly, despite the high mutation supply provided by mutators [G], no blaTEM-1 resistance mutations were found when the gene was located in the chromosome. By contrast, double and triple mutants readily emerged when the bla ${ }_{\mathrm{TEM}-1}$ gene was located on a plasmid ${ }^{66}$. Similarly, experimental evolution of resistance to a different $\beta$-lactam antibiotic (ceftazidime) showed that emergence of high-level resistance is contingent on the presence of the bla TEM-1 allele on a plasmid and it is not accessible when the gene is located on the chromosome $^{40}$. These results can be explained by the interplay of two direct consequences of the multi-copy nature of plasmids. First, plasmid genes offer a large mutational target due to their high copy number, and thus beneficial mutations will occur at higher frequencies (that is, more mutations per gene) than in chromosomal genes. This effect is simple to reconcile with classical evolutionary theory: increasing the rate at which beneficial mutations are introduced into populations increases the rate of adaptation ${ }^{67}$. Second, $\mathrm{PCN}$ amplifies the gene dosage of new mutations, and 
their expression levels are rapidly maximised at the population level. As stated before, resistance levels conferred by $\beta$-lactamases drastically depend on their gene dosage $^{40,56}$, and thus mutations improving blaTEM-1 activity confer higher resistance levels when they are encoded on plasmids rather than on chromosomes ${ }^{40}$. Similarly, directed evolution experiments, in which plasmids containing a gene of interest are mutagenized, have shown that mutations that increase PCN are positively selected alongside mutations that directly improve the catalytic activity of the gene of interest $^{68}$. Together, these results demonstrate that $\mathrm{PCN}$ not only contributes to optimizing existing traits but also facilitates the evolution of new biochemical activities, and that gene amplification and mutation - two of the dominant mechanisms of bacterial evolution - are tightly linked due to the multi-copy nature of plasmids.

Bacterial plasmids are prone to recombination. First, the high density of repeated sequences on plasmids, such as those associated with transposable elements [G], creates recombinational hot-spots that lead to a high rate of en bloc deletions and capture of new genetic elements from distinct sources. In fact, more than $40 \%$ of plasmids deposited in the NCBI database are mosaic plasmids that carry substantial fragments of other plasmids ${ }^{69}$. A particularly revealing example comes from the plasmids of the phylum Firmicutes, which are characterised by their recombinationdriven modularity. These plasmids show a high degree of mosaicism, often including multiple origins of replication within the same plasmid backbone ${ }^{70}$. Second, host defence systems cause an increase in plasmid recombination as a side-effect. Host defences, such as CRISPR-Cas and restriction-modification systems, typically prevent plasmid establishment by producing double-strand breaks in foreign DNA ${ }^{71}$. Double-strand breaks, in turn, promote plasmid recombination, as they are the preferred substrate of most bacterial recombinases. Some plasmids are equipped 
with dedicated genes to repair double-strand breaks through recombination ${ }^{72}$, suggesting that recombinational evasion of host defences might a be a common strategy among plasmids.

Recombination has had a major role in the evolution of plasmid-associated antibiotic resistance genes such as $\beta$-lactamases ${ }^{73,74}$ and fluoroquinolone resistance $q n r$ genes (which protect DNA gyrase and topoisomerase IV from fluoroquinolone binding ${ }^{75}$ ). The evolution of these gene families is characterized by frequent recombination between different alleles, combining mutations that had emerged separately ${ }^{73,75}$. At a broader scale, recombination can create chimeric plasmids that contain antibiotic resistance cassettes from different plasmids, resulting in multi-resistance replicons that pose a great clinical concern ${ }^{76-79}$. For instance, non-conjugative plasmids carrying the colistin resistance gene mcr-1 have been shown to recombine with conjugative plasmids carrying additional resistance determinants such as the bla $a_{\mathrm{NDM}}$ and blaстх-м $\beta$-lactamases ${ }^{76,77}$. Importantly, these multi-resistance plasmid hybrids are stably maintained, and spread through conjugation among bacterial populations ${ }^{76,77}$. Plasmid recombination occurs more frequently in pathogenic $E$. coli strains than in their commensal counterparts ${ }^{64}$, suggesting that chimeric plasmids might be more common among the former. Additionally, several plasmid families are known to engage in plasmid-mediated gene capture, also known as retrotransfer, by which plasmids are able to transfer to a new host, incorporate genomic fragments of the recipient, and be transferred back to the original donor ${ }^{13}$.

Plasmid recombination has driven the evolution of IncP-1, IncW and IncF plasmid families by increasing plasmid fitness through capture and combination of different genes, facilitating their adaptation to different hosts ${ }^{80-83}$. Other paradigmatic examples come from the agrobacterial (tumour-inducing) $\mathrm{Ti}$ and (root-inducing) $\mathrm{Ri}$ plasmids. The presence of $\mathrm{Ti}$ and Ri plasmids in Agrobacterium species drives their 
transition from non-pathogenic bacteria to tumour-inducing plant pathogens ${ }^{84}$. These plasmids descend from only a handful of parental lineages and are remarkably conserved ${ }^{85}$. However, extensive recombination has reshuffled functional modules between distinct lineages, promoting their diversification and facilitating their massive spread in agricultural settings ${ }^{85}$.

Recombination also affects plasmid evolution on shorter time scales. For instance, IncQ plasmids replicate through a strand displacement mechanism that renders them particularly prone to recombination ${ }^{86}$. Once an IncQ plasmid arrives in a new host, recombination rapidly creates variants with smaller sizes that can lack both accessory and essential plasmid genes. Interestingly, these streamlined variants compete for intracellular replication with the full-length version of the plasmid, reducing its $\mathrm{PCN}$ and the fitness burden associated with it ${ }^{87}$. This is potentially beneficial for the host, as it can maintain burdensome, but potentially beneficial plasmid genes at a low physiological $\operatorname{cost}^{87}$. This example, and others ${ }^{88,89}$, highlight the role of recombination in streamlining plasmid genomes to reduce the cost of plasmid maintenance. Together, these studies suggest that plasmids, owing to their copy number, gene content, and replication modes are particularly prone to engaging in recombination, which in turn promotes plasmid evolution.

\section{[H2] Complex population dynamics of plasmid-encoded alleles}

One important consequence of the multi-copy nature of plasmids is that once a novel allele arises on a plasmid-encoded locus, it will be initially present in only one of the plasmid copies. Therefore, plasmids carrying novel alleles will coexist with plasmid copies carrying the ancestral allele until they are eventually segregated into distinct cell lineages. The coexistence of distinct variants of the same plasmid is termed heteroplasmy [G] ${ }^{90}$. In other words, heteroplasmid cells carry heterozygous plasmid alleles whereas homoplasmid cells carry homozygous plasmid alleles ${ }^{90}$. 
Heteroplasmy poses consequences for plasmid evolution that are reminiscent of classical population genetics (FIG. 2).

First, because segregation of mutant and non-mutant plasmids copies is random, daughter cells often inherit a different allelic composition than that of the parental cell. Plasmid evolution is thus subjected to an extra layer of genetic drift [G], known as segregational drift, by which intracellular frequencies of plasmid-encoded alleles change due to random inheritance ${ }^{91}$. Segregational drift reduces the establishment probability of plasmid neutral alleles and increases the fixation time of plasmid beneficial mutations compared to chromosomal mutations ${ }^{91}$ (FIG. 2). In fact, experimental evolution under selective conditions for plasmid maintenance during 800 generations showed that HCPs accumulate less mutations than expected by chance due to segregational drift ${ }^{91}$. Segregational drift might help explain the striking conservation in the core genomes of some plasmid families that show worldwide distribution, such as the broad-host range IncP-1 family ${ }^{92}$.

If a beneficial mutation emerges in a plasmid copy before a former mutation in a different copy reaches fixation, a second effect termed plasmid interference takes place. Plasmid interference produces an analogous effect to clonal interference [G], but at the cellular level instead of at the population level. During plasmid interference, two (or more) beneficial mutations coexist and compete for establishment in the plasmid pool of a single cell lineage under heteroplasmy ${ }^{88}$. One study evolved populations of $E$. coli carrying costly versions of the chloramphenicol resistance cat gene in a HCP. Sequencing of evolved clonal populations showed two to three coexisting beneficial mutations in the cat gene competing for fixation at the cellular level. This plasmid interference translated into population-level polymorphisms that were maintained over large time-scales ${ }^{88}$. Therefore, as in clonal interference, plasmid interference delays the fixation of beneficial mutations in plasmid populations 
and can even lead to the loss of beneficial mutations ${ }^{88}$. However, recombination between plasmid variants is expected to frequently assort competing mutations in the same plasmid backbone ${ }^{73}$, modulating the impact of plasmid interference on the evolution of plasmid encoded traits.

Due to the factors mentioned above, plasmid mutations experience considerably longer fixation times than chromosomal mutations ${ }^{91,93,94}$. This negatively affects the establishment probability of novel plasmid alleles, yet it also implies that plasmid loci remain polymorphic for dozens of bacterial generations. Therefore, by shielding mutations from selection, plasmids provide a source of standing genetic variation [G] 96. Upon environmental change, standing genetic variation allows bacterial populations to adapt through pre-existing mutations, and occurs faster than adaptation through de novo mutations ${ }^{95}$. Growing populations of bacteria accumulate mutations randomly, but selection and drift rapidly reduce the frequency of chromosomal mutations in the population. In plasmids, these polymorphisms are maintained over larger timescales, which increases the chances of bacterial populations escaping extinction caused by sudden environmental changes such as antibiotic treatment ${ }^{94}$.

From a different perspective, when a gene evolves to gain a new function, it often does so at the expense of its original function. These negative correlations between ancestral and novel activities are called trade-offs [G] and are a pervasive constraint in protein evolution ${ }^{96}$. Trade-offs occur because beneficial mutations in one environment are often deleterious if the context changes. For instance, evolution of antibiotic resistance is dominated by trade-offs in which a mutation confers resistance to a new antibiotic, but at the expense of a reduced activity against the antibiotic to which it originally conferred resistance ${ }^{97}$. For example, the bla TEM-1 $\beta-$ lactamase confers high level resistance to penicillin-derived antibiotics such as 
ampicillin, but has negligible activity against third-generation cephalosporins such as ceftazidime. Evolution of bla $\mathrm{TEM}_{\mathrm{T}-1}$ towards ceftazidime resistance has been shown to occur predominantly through the acquisition of a single mutation that gives rise to the blatEM-12 allele $^{97}$. The evolved allele confers high-level resistance to ceftazidime, but does so at the expense of reducing its activity against ampicillin. As a result, when both antibiotics are present either simultaneously or alternatively during fluctuating selective pressures, trade-offs limit the evolution of antibiotic resistance ${ }^{97}$. However, when those alleles are plasmid-encoded, they co-exist within the cell for hundreds of bacterial generations under heteroplasmy, relieving the constraints imposed by tradeoffs in the evolution of antibiotic resistance ${ }^{98}$. Plasmids therefore represent excellent scaffolds for the evolution of novel functions because they can maintain genetic diversity both intracellularly and at the population level ${ }^{98}$. Interestingly, the plasmidlike genomes of bacterium-derived eukaryotic organelles also overcame evolutionary trade-offs by accumulating genetic variation in the form of long-lasting heteroplasmy. For instance, the mitochondrial genome of pill-bugs (Armadillidium vulgare) shows a conserved polymorphism in their alanine and valine alloaceptor tRNA that has persisted for more than thirty million years ${ }^{99}$.

\section{[H2] The role of genetic dominance}

A given mutation can have profoundly different phenotypic effects depending on whether it occurs on a chromosomal or plasmid gene. Besides the effects on gene expression levels discussed above, the phenotypic effect of plasmid mutations will depend on their degree of genetic dominance. Genetic dominance is the relationship between alleles of the same gene, in which the phenotypic contribution of one allele (termed recessive) is masked by the presence of a second allele (dominant). However, it can occur that one of the alleles is not completely dominant over the other. In these cases, both alleles are manifested either simultaneously (termed co- 
dominance) or as a blend of the phenotype of both alleles (incomplete dominance). The notion of genetic dominance dates back to Gregor Mendel and is considered to drive the evolution of polyploid and diploid organisms. This is because recessive mutations are less likely to contribute to the phenotype than dominant mutations, and as a consequence there is a bias against the establishment of recessive beneficial mutations in polyploid organisms, an effect known as Haldane's sieve ${ }^{100}$. As most bacteria have been assumed to carry a unique copy of their chromosomes ${ }^{49}$, the effect of genetic dominance has traditionally been neglected in bacterial evolution (but see REF. ${ }^{101}$ where the effect of genetic dominance on the fate of chromosomal mutations is explored). However, genetic dominance has far reaching consequences for plasmid evolution ${ }^{94,102}$.

Recessive plasmid alleles will be unable to contribute to phenotypes as long as they co-exist with wild-type alleles within the same cell (FIG. 3). As explained above, plasmids alleles can stably co-exist for dozens of generations under heteroplasmy, and thus genetic dominance should have a key role on determining the establishment probability of novel plasmid mutations ${ }^{94,102}$. Genetic dominance has two major implications for plasmid evolution. First, plasmids should evolve mainly through dominant mutations and therefore have fewer mutations available than bacterial chromosomes (which evolve through both dominant and recessive mutations $)^{94,102}$. In fact, the rate of mutations conferring antibiotic resistance was found to be 3 to 10 times lower in plasmids than in chromosomes when the available mutations were recessive, and 4 times higher when the selected mutations were dominant ${ }^{94,102}$. Second, genetic dominance appears to dictate the fate of horizontally transferred alleles if the recipient bacterium already carries an allele of the transferred gene on its chromosome ${ }^{94,102}$. This was demonstrated experimentally by showing that conjugative transfer of a recessive, quinolone resistant allele of the gyrA 
gene provided no resistance in the recipient cell ${ }^{102}$, as a wild-type version of this housekeeping gene is naturally present on most bacterial chromosomes. Genetic redundancy between plasmids and chromosomes is likely to be common, considering that plasmids and chromosomes frequently share genetic content $^{26,82,83,103,104}$, and that plasmid transfer typically occurs between close relatives $^{105}$. Although the pervasiveness of the effects of genetic dominance in bacterial evolution are yet to be established, the interplay between the aforementioned two effects should shape the genetic repertoire of plasmids. In fact, bioinformatic evidence suggests that recessive alleles of antibiotic resistance genes tend to be under-represented on plasmids and other mobile genetic elements in comparison to chromosomes ${ }^{102}$. This might have important implications for bacterial evolution, but also for human health, as it offers a framework to predict which antibiotic resistance genes are likely to be mobilised by plasmids and other mobile genetic elements (Box 3).

\section{[H1] Further plasmid effects on evolution}

Beyond the effects associated with the multi-copy nature of plasmids, other plasmid properties may also contribute to accelerate bacterial evolution. During conjugation, plasmids are transferred as single stranded DNA, which in turn activates the bacterial SOS stress response [G] ${ }^{106}$. The SOS response coordinates the expression of dozens of bacterial genes involved in DNA repair and cell cycle control, and it is known to fuel bacterial evolvability through an increase in recombination and mutagenesis $^{107}$. For instance, conjugation of antibiotic resistance plasmids from $E$. coli to Vibrio cholerae strongly induces the SOS response, leading to genomic rearrangements mediated by the site-specific integron [G] integrase ${ }^{106}$. 
A large proportion of conjugative plasmids encode SOS-regulated genes that directly enhance mutagenesis ${ }^{108,109}$. Most of these plasmids encode functional homologues of chromosomal UmuDC proteins. The $u m u D C$ operon encodes an error-prone DNA polymerase, which is capable of replicating heavily damaged DNA templates, but does so at a reduced fidelity. Expression of plasmid umuDC genes is tightly controlled as part of the bacterial SOS response and leads to transient mutagenesis upon plasmid transfer ${ }^{110}$. For instance, rizhobial symbiotic plasmids encode complex traits that mediate the relationship between legume plants and bacteria ${ }^{23}$. These plasmids frequently encode UmuDC homologues that boost the mutation rate of the bacterial host upon entry ${ }^{111}$. Experimental replay of rizhobium evolution has shown that co-transfer of UmuDC with symbiotic genes on a plasmid accelerates the transition of plant pathogens into legume symbionts by generating a burst of genome diversity after transiently increasing the chromosomal mutation rate ${ }^{111}$. Thus, SOSmediated mutagenesis is not restricted to plasmid DNA and also affects chromosomal DNA, promoting the evolution of the bacterial host ${ }^{111}$. Additionally, it has been hypothesized that plasmid-mediated SOS induction might also serve to accelerate the adaptation of the bacterial host to incoming plasmids, and thus might be particularly exploited by broad-host range plasmids ${ }^{106} \square$. In agreement with this hypothesis, narrow-host range plasmids often carry dedicated genes to prevent SOS induction ${ }^{106}$.

Plasmids also facilitate the evolution of their bacterial host through mechanisms independent of SOS induction. For instance, non-growing populations of $E$. coli have been shown to experience increased rates of mutagenesis. This mutagenesis, which seems to be restricted to plasmid genomes, occurs through an increase in PCN in a subpopulation of cells. Conjugative transfer of these plasmids between clone mates 
further amplifies plasmid genomes, which are then subject to extensive mutagenesis mediated by the plasmid-encoded, error-prone polymerase $\operatorname{DinB}^{47,112}$.

Additionally, plasmids are often associated with other mobile genetic elements, such as transposable elements and integrons. Once a plasmid arrives in a new host, these elements commonly transfer to the chromosome, where they foster bacterial adaptation 26,104 . For instance, transposons encoding mercury resistance were shown to transfer between distinct Pseudomonas species using conjugative plasmids as vehicles. The transposons were able to mobilize from chromosomes to plasmids, and back to chromosomes, disseminating mercury resistance among soil communities and allowing their hosts to colonize mercury polluted environments ${ }^{26}$.

Finally, epistatic interactions [G] have been shown to occur between chromosomal and plasmid genes ${ }^{113,114}$. As a consequence, plasmids can alter host evolution by changing the selective effects of chromosomal mutations. For example, epistatic interactions between conjugative plasmids and chromosomal mutations alter the evolutionary paths that $E$. coli follows to evolve antibiotic resistance ${ }^{113}$. Antibiotic resistance chromosomal mutations are usually costly to the bacterial host. However, epistasis between plasmids and chromosomal mutations has been shown to reduce the cost of resistance, and therefore an initially deleterious mutation may become beneficial upon plasmid acquisition ${ }^{113}$. Therefore, these epistatic interactions might pave the way for the emergence of multidrug resistant bacteria at very low fitness costs. Additionally, epistasis often occurs between genes that have similar functional roles $^{115}$, and we speculate that chromosome-plasmid epistasis should be especially pronounced for traits like antibiotic resistance and metabolism that are prevalent on both plasmids and chromosomes. 


\section{[H1] Conclusions and future perspectives}

Despite 80 years of extensive study of plasmid biology, several outstanding questions remain to be answered (Box 4). The recent advances reviewed here highlight that plasmids are likely to evolve differently than bacterial chromosomes, and that plasmids create a scenario in which opposing forces determine the evolution of plasmid-encoded traits. On the one hand, plasmids generate genetic variability at higher rates than bacterial chromosomes, show dedicated mechanisms to increase their evolvability, and can store genetic variation for hundreds of bacterial generations. On the other hand, genetic dominance, plasmid interference and segregational drift may limit the evolutionary potential of plasmids. This leads to the question of whether plasmids evolve faster than bacterial chromosomes. Several lines of evidence suggest that this is indeed the case. First, experimental evolution has shown that plasmids accelerate evolution when selective pressure is high ${ }^{40,66}$. Second, mathematical models predict that plasmids will increase the rate of beneficial mutations as long as these mutations are dominant or have strong gene dosage effects ${ }^{40,94,102}$. Third, plasmids are enriched in dispensable genes for the bacterial host. This dispensability should reduce purifying selection [G], allowing plasmids to accumulate genetic variability during evolutionary relevant time scales ${ }^{116}$. Fourth, recombination is frequent among plasmid genomes. The genetic plasticity provided by recombination is further enhanced because plasmids frequently engage in HGT, and thus they cohabit with a wide variety of bacterial genetic backgrounds. This increases the repertoire of beneficial variants that can recombine with plasmid genomes, speeding up the evolution of plasmid-encoded traits ${ }^{117}$. Altogether, these evidences suggest that plasmids might act as evolutionary catalysts, promoting their own evolution and that of their bacterial host through a plethora of distinct mechanisms that go above and beyond HGT. It is our view that gaining a better 
understanding of plasmid evolution will shed light on the mechanisms that fuel bacterial diversity and help explain the extreme ecological success of prokaryotes.

\section{References}

1. Lederberg, J. Cell genetics and hereditary symbiosis. Physiol. Rev. 32, 403430 (1952).

\section{A landmark paper in which the term 'plasmid' is proposed for the first time.}

2. Lederberg, J. Personal perspective: Plasmid (1952-1997). Plasmid vol. 39 1-9 (1998).

3. Werren, J. H. Selfish genetic elements, genetic conflict, and evolutionary innovation. Proc. Natl. Acad. Sci. U. S. A. 108, 10863-10870 (2011).

4. Rankin, D. J., Rocha, E. P. C. \& Brown, S. P. What traits are carried on mobile genetic elements, and why. Heredity vol. 106 1-10 (2011).

5. Hernández-Arriaga, A. M., Chan, W. T., Espinosa, M. \& Díaz-Orejas, R. Conditional Activation of Toxin-Antitoxin Systems: Postsegregational Killing and Beyond. Microbiol. Spectr. 2, (2014).

6. Norman, A., Hansen, L. H. \& Sørensen, S. J. Conjugative plasmids: Vessels of the communal gene pool. Philosophical Transactions of the Royal Society B: Biological Sciences vol. 364 2275-2289 (2009).

7. Ramsay, J. P. et al. An updated view of plasmid conjugation and mobilization in Staphylococcus . Mob. Genet. Elements 6, e1208317 (2016).

8. Carattoli, A. et al. Identification of plasmids by PCR-based replicon typing. J. Microbiol. Methods 63, 219-228 (2005).

9. Smillie, C., Garcillán-Barcia, M. P., Francia, M. V., Rocha, E. P. C. \& de la Cruz, F. Mobility of plasmids. Microbiol. Mol. Biol. Rev. 74, 434-52 (2010).

\section{A thorough analysis of plasmid mobility that sets the basis for mobility (MOB) typing.}

10. Douarre, P.-E., Mallet, L., Radomski, N., Felten, A. \& Mistou, M.-Y. Analysis of COMPASS, a New Comprehensive Plasmid Database Revealed Prevalence of Multireplicon and Extensive Diversity of IncF Plasmids. Front. Microbiol. 11, $1-15(2020)$. 
11. Acman, M., van Dorp, L., Santini, J. M. \& Balloux, F. Large-scale network analysis captures biological features of bacterial plasmids. Nat. Commun. 11, 2452 (2020).

Comprehensive analysis of plasmid whole-genome sequences using similarity networks that paves the way for a novel plasmid classification schemes.

12. Redondo-Salvo, S. et al. Pathways for horizontal gene transfer in bacteria revealed by a global map of their plasmids. Nat. Commun. 11, 3602 (2020).

13. Szpirer, C., Top, E., Couturier, M. \& Mergeay, M. Retrotransfer or gene capture: A feature of conjugative plasmids, with ecological and evolutionary significance. Microbiology 145, 3321-3329 (1999).

14. Tock, M. R. \& Dryden, D. T. F. The biology of restriction and anti-restriction. Curr. Opin. Microbiol. 8, 466-472 (2005).

15. Pinilla-Redondo, R. et al. Type IV CRISPR-Cas systems are highly diverse and involved in competition between plasmids. Nucleic Acids Res. 48, 20002012 (2020).

16. Ares-Arroyo, M. et al. PCR-Based Analysis of ColE1 Plasmids in Clinical Isolates and Metagenomic Samples Reveals Their Importance as Gene Capture Platforms. Front. Microbiol. 9, 469 (2018).

17. Attéré, S. A., Vincent, A. T., Paccaud, M., Frenette, M. \& Charette, S. J. The role for the small cryptic plasmids as moldable vectors for genetic innovation in Aeromonas salmonicida subsp. salmonicida. Front. Genet. 8, 1-11 (2017).

18. Pilla, G. \& Tang, C. M. Going around in circles: Virulence plasmids in enteric pathogens. Nat. Rev. Microbiol. 16, 484-495 (2018).

19. Carattoli, A. Resistance plasmid families in Enterobacteriaceae. Antimicrobial Agents and Chemotherapy vol. 53 2227-2238 (2009).

20. Brinkmann, H., Göker, M., Koblížek, M., Wagner-Döbler, I. \& Petersen, J. Horizontal operon transfer, plasmids, and the evolution of photosynthesis in Rhodobacteraceae. ISME J. 12, 1994-2010 (2018).

21. Manzano-Marín, A. et al. Serial horizontal transfer of vitamin-biosynthetic genes enables the establishment of new nutritional symbionts in aphids' disymbiotic systems. ISME J. 14, 259-273 (2020).

22. Anda, M. et al. Bacterial clade with the ribosomal RNA operon on a small plasmid rather than the chromosome. Proc. Natl. Acad. Sci. U. S. A. 112, 14343-14347 (2015).

An example of a HCP carrying an essential core gene for its bacterial host. 
23. Wang, $X$. et al. Comparative symbiotic plasmid analysis indicates that symbiosis gene ancestor type affects plasmid genetic evolution. Lett. Appl. Microbiol. 67, 22-31 (2018).

24. Soucy, S. M., Huang, J. \& Gogarten, J. P. Horizontal gene transfer: Building the web of life. Nat. Rev. Genet. 16, 472-482 (2015).

25. Vos, M., Hesselman, M. C., te Beek, T. A., van Passel, M. W. J. \& EyreWalker, A. Rates of Lateral Gene Transfer in Prokaryotes: High but Why? Trends in Microbiology vol. 23 598-605 (2015).

26. Hall, J. P. J., Williams, D., Paterson, S., Harrison, E. \& Brockhurst, M. A. Positive selection inhibits gene mobilization and transfer in soil bacterial communities. Nat. Ecol. Evol. 1, 1348-1353 (2017).

Elegant study that highlights the role of HGT in general and transposons in particular in the evolution of bacterial soil communities.

27. Bethke, J. H. et al. Environmental and genetic determinants of plasmid mobility in pathogenic Escherichia coli. Sci. Adv. 6, (2020).

28. Sørensen, S. J., Bailey, M., Hansen, L. H., Kroer, N. \& Wuertz, S. Studying plasmid horizontal transfer in situ: A critical review. Nat. Rev. Microbiol. 3, 700-710 (2005).

29. Leon-Sampedro, R. et al. Dissemination routes of the carbapenem resistance plasmid pOXA-48 in a hospital setting. bioRxiv 2020.04.20.050476 (2020) doi:10.1101/2020.04.20.050476.

30. Gumpert, H. et al. Transfer and Persistence of a Multi-Drug Resistance Plasmid in situ of the Infant Gut Microbiota in the Absence of Antibiotic Treatment. Front. Microbiol. 8, 1852 (2017).

31. Porse, A. et al. Genome dynamics of Escherichia coli during antibiotic treatment: Transfer, loss, and persistence of genetic elements in situ of the infant gut. Front. Cell. Infect. Microbiol. 7, (2017).

32. Munck, C., Sheth, R. U., Freedberg, D. E. \& Wang, H. H. Recording mobile DNA in the gut microbiota using an Escherichia coli CRISPR-Cas spacer acquisition platform. Nat. Commun. 11, 1-11 (2020).

33. Thomas, C. M. \& Summers, D. Bacterial Plasmids. Encycl. Life Sci. 1-9 (2008) doi:10.1002/9780470015902.a0000468.pub2.

34. Summers, D. K. Plasmid Replication and its Control. in The Biology of Plasmids 31-64 (Blackwell Publishing Ltd., 2009). doi:10.1002/9781444313741.ch3.

35. Wong Ng, J., Chatenay, D., Robert, J. \& Poirier, M. G. Plasmid copy number noise in monoclonal populations of bacteria. Phys. Rev. E - Stat. Nonlinear, Soft Matter Phys. 81, (2010). 
36. Münch, K., Münch, R., Biedendieck, R., Jahn, D. \& Müller, J. Evolutionary model for the unequal segregation of high copy plasmids. PLoS Comput. Biol. 15, (2019).

37. Nordström, K. Plasmid R1-Replication and its control. Plasmid vol. 55 1-26 (2006).

38. Jahn, M., Vorpahl, C., Hübschmann, T., Harms, H. \& Müller, S. Copy number variability of expression plasmids determined by cell sorting and Droplet Digital PCR. Microb. Cell Fact. 15, 211 (2016).

39. Mei, $\mathrm{H}$. et al. A High-Resolution View of Adaptive Event Dynamics in a Plasmid. Genome Biol. Evol. 11, 3022-3034 (2019).

40. San Millan, A., Escudero, J. A., Gifford, D. R., Mazel, D. \& MacLean, R. C. Multicopy plasmids potentiate the evolution of antibiotic resistance in bacteria. Nat. Ecol. Evol. 1, 0010 (2016).

First paper to show that plasmids increase evolvability of antibiotic resistance.

41. Santos-Lopez, A. et al. A naturally occurring SNP in plasmid pB1000 produces a reversible increase in antibiotic resistance. Antimicrob. Agents Chemother. 2, AAC.01735-16 (2016).

42. San Millan, A. et al. Small-plasmid-mediated antibiotic resistance is enhanced by increases in plasmid copy number and bacterial fitness. Antimicrob. Agents Chemother. 59, 3335-3341 (2015).

43. Thompson, M. G. et al. Isolation and characterization of novel mutations in the pSC101 origin that increase copy number. Sci. Rep. 8, 1590 (2018).

44. Cho, H. \& Winans, S. C. VirA and VirG activate the Ti plasmid repABC operon, elevating plasmid copy number in response to wound-released chemical signals. Proc. Natl. Acad. Sci. U. S. A. 102, 14843-14848 (2005).

45. Wang, H. et al. Increased plasmid copy number is essential for Yersinia T3SS function and virulence. Science (80-. ). 353, 492-495 (2016).

46. Akasaka, N. et al. Change in the plasmid copy number in acetic acid Bacteria in response to growth phase and acetic acid Concentration. J. Biosci. Bioeng. 119, 661-668 (2015).

47. Sano, E., Maisnier-Patin, S., Aboubechara, J. P., Quiñones-Soto, S. \& Roth, J. R. Plasmid copy number underlies adaptive mutability in bacteria. Genetics 198, 919-933 (2014).

48. Pappas, K. M. \& Winans, S. C. A LuxR-type regulator from Agrobacterium tumefaciens elevates $\mathrm{Ti}$ plasmid copy number by activating transcription of plasmid replication genes. Mol. Microbiol. 48, 1059-1073 (2003). 
49. Pecoraro, V., Zerulla, K., Lange, C. \& Soppa, J. Quantification of ploidy in proteobacteria revealed the existence of monoploid, (mero-)oligoploid and polyploid species. PLoS One 6, (2011).

50. Klumpp, S. Growth-Rate Dependence Reveals Design Principles of Plasmid Copy Number Control. PLoS One 6, e20403 (2011).

51. Atlung, T., Christensen, B. B. \& Hansen, F. G. Role of the rom protein in copy number control of plasmid pBR322 at different growth rates in Escherichia coli K-12. Plasmid 41, 110-119 (1999).

52. San Millan, A. et al. Integrative analysis of fitness and metabolic effects of plasmids in Pseudomonas aeruginosa PAO1. ISME J. 12, 3014-3024 (2018).

53. Park, C. \& Zhang, J. High expression hampers horizontal gene transfer. in Genome Biology and Evolution vol. 4 523-532 (2012).

54. Condon, C., Liveris, D., Squires, C., Schwartz, I. \& Squires, C. L. rRNA operon multiplicity in Escherichia coli and the physiological implications of rrn inactivation. J. Bacteriol. 177, 4152-4156 (1995).

55. Ikeda, S. et al. Community shifts of soybean stem-associated bacteria responding to different nodulation phenotypes and $\mathrm{N}$ levels. ISME J. 4, 315326 (2010).

56. Shen, Z. et al. Increased Plasmid Copy Number Contributes to the Elevated Carbapenem Resistance in OXA-232-Producing Klebsiella pneumoniae. Microb. Drug Resist. mdr.2018.0407 (2019) doi:10.1089/mdr.2018.0407.

57. El-Halfawy, O. M. \& Valvano, M. A. Antimicrobial heteroresistance: An emerging field in need of clarity. Clinical Microbiology Reviews vol. 28 191207 (2015).

58. Andersson, D. I., Nicoloff, H. \& Hjort, K. Mechanisms and clinical relevance of bacterial heteroresistance. Nat. Rev. Microbiol. 17, 479-496 (2019).

59. Nicoloff, H., Hjort, K., Levin, B. R. \& Andersson, D. I. The high prevalence of antibiotic heteroresistance in pathogenic bacteria is mainly caused by gene amplification. Nat. Microbiol. 4, 504-514 (2019).

60. Cascales, E. et al. Colicin biology. Microbiol. Mol. Biol. Rev. 71, 158-229 (2007).

61. Bayramoglu, B. et al. Bet-hedging in bacteriocin producing Escherichia coli populations: The single cell perspective. Sci. Rep. 7, (2017).

62. Mrak, P., Podlesek, Z., Van Putten, J. P. M. \& Žgur-Bertok, D. Heterogeneity in expression of the Escherichia coli colicin K activity gene cka is controlled by the SOS system and stochastic factors. Mol. Genet. Genomics 277, 391-401 (2007). 
63. Tomanek, I. et al. Gene amplification as a form of population-level gene expression regulation. Nat. Ecol. Evol. 4, 612-625 (2020).

64. Rodríguez-Beltrán, J. et al. High recombinant frequency in extraintestinal pathogenic escherichia coli strains. Mol. Biol. Evol. 32, 1708-1716 (2015).

65. Niaudet, B., Jannière, L. \& Ehrlich, S. D. Recombination between repeated DNA sequences occurs more often in plasmids than in the chromosome of Bacillus subtilis. MGG Mol. Gen. Genet. 197, 46-54 (1984).

66. Couce, A., Rodríguez-Rojas, A. \& Blázquez, J. Bypass of genetic constraints during mutator evolution to antibiotic resistance. Proc. R. Soc. B Biol. Sci. 282, 20142698 (2015).

67. De Visser, J. A. G. M., Zeyl, C. W., Gerrish, P. J., Blanchard, J. L. \& Lenski, R. E. Diminishing returns from mutation supply rate in asexual populations. Science (80-. ). 283, 404-406 (1999).

68. Million-Weaver, S., Alexander, D. L., Allen, J. M. \& Camps, M. Quantifying plasmid copy number to investigate plasmid dosage effects associated with directed protein evolution. Methods Mol. Biol. 834, 33-48 (2012).

69. Pesesky, M. W., Tilley, R. \& Beck, D. A. C. Mosaic plasmids are abundant and unevenly distributed across prokaryotic taxa. Plasmid 102, 10-18 (2019).

70. Lanza, V. F., Tedim, A. P., Martínez, J. L., Baquero, F. \& Coque, T. M. The Plasmidome of Firmicutes: Impact on the Emergence and the Spread of Resistance to Antimicrobials. Microbiol. Spectr. 3, (2015).

71. Bernheim, A. \& Sorek, R. The pan-immune system of bacteria: antiviral defence as a community resource. Nat. Rev. Microbiol. 18, 113-119 (2020).

72. Roy, D., Huguet, K. T., Grenier, F. \& Burrus, V. IncC conjugative plasmids and SXT/R391 elements repair double-strand breaks caused by CRISPR-Cas during conjugation. Nucleic Acids Res. 1-13 (2020) doi:10.1093/nar/gkaa518.

73. Barlow, M., Fatollahi, J. \& Salverda, M. Evidence for recombination among the alleles encoding TEM and SHV $\beta$-lactamases. J. Antimicrob. Chemother. 63, 256-259 (2009).

74. Mroczkowska, J. E. \& Barlow, M. Recombination and selection can remove blaTEM alleles from bacterial populations. Antimicrob. Agents Chemother. 52, 3408-3410 (2008).

75. Baquirin, M. H. C. \& Barlow, M. Evolution and recombination of the plasmidic qnr alleles. J. Mol. Evol. 67, 103-110 (2008).

76. Xie, M., Li, R., Liu, Z., Chan, E. W. C. \& Chen, S. Recombination of plasmids in a carbapenem-resistant NDM-5-producing clinical Escherichia coli isolate. J. Antimicrob. Chemother. 73, 1230-1234 (2018). 
77. He, D. et al. Emergence of a hybrid plasmid derived from IncN1-F33:A-:B- And mcr-1-bearing plasmids mediated by IS26. J. Antimicrob. Chemother. 74, 3184-3189 (2019).

78. Li, $\mathrm{Y}$. et al. Evidence of Illegitimate Recombination Between Two Pasteurellaceae Plasmids Resulting in a Novel Multi-Resistance Replicon, pM3362MDR, in Actinobacillus pleuropneumoniae. Front. Microbiol. 9, 2489 (2018).

79. Desmet, $\mathrm{S}$. et al. Antibiotic resistance plasmids cointegrated into a megaplasmid harboring the bla OXA-427 carbapenemase gene. Antimicrob. Agents Chemother. 62, (2018).

80. Fidelma Boyd, E., Hill, C. W., Rich, S. M. \& Hard, D. L. Mosaic structure of plasmids from natural populations of Escherichia coli. Genetics 143, 10911100 (1996).

81. Norberg, P., Bergström, M., Jethava, V., Dubhashi, D. \& Hermansson, M. The IncP-1 plasmid backbone adapts to different host bacterial species and evolves through homologous recombination. Nat. Commun. 2, 1-11 (2011).

82. Fernández-López, R. et al. Dynamics of the IncW genetic backbone imply general trends in conjugative plasmid evolution. FEMS Microbiology Reviews vol. 30 942-966 (2006).

83. Osborn, A. M., da Silva Tatley, F. M., Steyn, L. M., Pickup, R. W. \& Saunders, J. R. Mosaic plasmids and mosaic replicons: Evolutionary lessons from the analysis of genetic diversity in IncFll-related replicons. Microbiology 146, 2267-2275 (2000).

84. Gordon, J. E. \& Christie, P. J. The Agrobacterium Ti Plasmids. Microbiol. Spectr. 2, (2014).

85. Weisberg, A. J. et al. Unexpected conservation and global transmission of agrobacterial virulence plasmids. Science (80-. ). 368, eaba5256 (2020).

86. Kuzminov, A. Single-strand interruptions in replicating chromosomes cause double-strand breaks. Proc. Natl. Acad. Sci. 98, 8241-8246 (2001).

87. Zhang, X., Deatherage, D. E., Zheng, H., Georgoulis, S. J. \& Barrick, J. E. Evolution of satellite plasmids can prolong the maintenance of newly acquired accessory genes in bacteria. Nat. Commun. 10, 1-12 (2019).

This elegant study highlights the role of recombination in plasmid evolution.

88. Bedhomme, S., Perez Pantoja, D. \& Bravo, I. G. Plasmid and clonal interference during post horizontal gene transfer evolution. Mol. Ecol. 26, 1832-1847 (2017). 
Describes for the first time the effects of plasmid interference in evolving bacterial populations.

89. Porse, A., Schønning, K., Munck, C. \& Sommer, M. O. A. Survival and Evolution of a Large Multidrug Resistance Plasmid in New Clinical Bacterial Hosts. Mol. Biol. Evol. 33, 2860-2873 (2016).

90. Novick, R. P. Plasmid incompatibility. Microbiol. Rev. 51, 381-395 (1987).

91. Ilhan, J. et al. Segregational Drift and the Interplay between Plasmid Copy Number and Evolvability. Mol. Biol. Evol. 36, 472-486 (2019).

Explores the role of segregational drift in plasmid evolution through a combined experimental and modelling approach.

92. Szczepanowski, R. et al. Sequencing and comparative analysis of IncP-1a antibiotic resistance plasmids reveal a highly conserved backbone and differences within accessory regions. J. Biotechnol. 155, 95-103 (2011).

93. Halleran, A. D., Flores-Bautista, E. \& Murray, R. M. Quantitative characterization of random partitioning in the evolution of plasmid-encoded traits. bioRxiv 594879 (2019) doi:10.1101/594879.

94. Santer, M. \& Uecker, H. Evolutionary Rescue and Drug Resistance on Multicopy Plasmids. Genetics genetics.303012.2019 (2020) doi:10.1534/genetics.119.303012.

95. Barrett, R. D. H. \& Schluter, D. Adaptation from standing genetic variation. Trends Ecol. Evol. 23, 38-44 (2008).

96. Soskine, M. \& Tawfik, D. S. Mutational effects and the evolution of new protein functions. Nat Rev Genet 11, 572-582 (2010).

97. Mroczkowska, J. E. \& Barlow, M. Fitness trade-offs in blaTEM evolution. Antimicrob. Agents Chemother. 52, 2340-2345 (2008).

98. Rodriguez-Beltran, J. et al. Multicopy plasmids allow bacteria to escape from fitness trade-offs during evolutionary innovation. Nat. Ecol. Evol. 2, 873-881 (2018).

Explores the role of plasmids as drivers of evolutionary innovation, highlighting their ability to maintain genetic diversity against strong selective forces.

99. Doublet, V., Souty-Grosset, C., Bouchon, D., Cordaux, R. \& Marcadé, I. A thirty million year-old inherited heteroplasmy. PLoS One 3, e2938 (2008).

100. Haldane, J. B. S. A Mathematical Theory of Natural and Artificial Selection, Part V: Selection and Mutation. Math. Proc. Cambridge Philos. Soc. 23, 838844 (1927). 
101. Sun, L. et al. Effective polyploidy causes phenotypic delay and influences bacterial evolvability. PLOS Biol. 16, e2004644 (2018).

102. Rodríguez-Beltrán, J. et al. Genetic dominance governs the evolution and spread of mobile genetic elements in bacteria. Proc. Natl. Acad. Sci. 202001240 (2020) doi:10.1073/pnas.2001240117.

First article to demonstrate that genetic dominance shapes plasmid evolution and genetic content.

103. Zheng, J. et al. Plasmids are vectors for redundant chromosomal genes in the Bacillus cereus group. BMC Genomics 16, 6 (2015).

104. Sheppard, A. E. et al. Nested Russian doll-like genetic mobility drives rapid dissemination of the carbapenem resistance gene blakpc. Antimicrob. Agents Chemother. 60, 3767-3778 (2016).

105. Dimitriu, T., Marchant, L., Buckling, A. \& Raymond, B. Bacteria from natural populations transfer plasmids mostly towards their kin. Proc. R. Soc. B Biol. Sci. 286, 20191110 (2019).

106. Baharoglu, Z., Bikard, D. \& Mazel, D. Conjugative DNA Transfer Induces the Bacterial SOS Response and Promotes Antibiotic Resistance Development through Integron Activation. PLoS Genet. 6, e1001165 (2010).

Elegantly demonstrates that the SOS system is induced in response to plasmid conjugative transfer and how this leads to genomic rearrangements.

107. Maslowska, K. H., Makiela-Dzbenska, K. \& Fijalkowska, I. J. The SOS system: A complex and tightly regulated response to DNA damage. Environ. Mol. Mutagen. 60, 368-384 (2019).

108. Howarth, S. Resistance to the bactericidal effect of ultraviolet radiation conferred on Enterobacteria by the colicine factor coli. J. Gen. Microbiol. 40, 43-55 (1965).

109. Strike, P. \& Lodwick, D. Plasmid genes affecting DNA repair and mutation. J. Cell Sci. SUPPL. 6, 303-321 (1987).

110. Woodgate, R. \& Sedgwick, S. G. Mutagenesis induced by bacterial UmuDC proteins and their plasmid homologues. Molecular Microbiology vol. 6 22132218 (1992).

111. Remigi, P. et al. Transient Hypermutagenesis Accelerates the Evolution of Legume Endosymbionts following Horizontal Gene Transfer. PLoS Biol. 12, e1001942 (2014).

112. Nguyen, A., Maisnier-Patin, S., Yamayoshi, I., Kofoid, E. \& Roth, J. R. Selective Inbreeding: Genetic Crosses Drive Apparent Adaptive Mutation in the Cairns-Foster System of Escherichia coli. Genetics 214, 333-354 (2020). 
113. Silva, R. F. et al. Pervasive Sign Epistasis between Conjugative Plasmids and Drug-Resistance Chromosomal Mutations. PLoS Genet. 7, e1002181 (2011).

114. Loftie-Eaton, W. et al. Evolutionary Paths That Expand Plasmid Host-Range: Implications for Spread of Antibiotic Resistance. Mol. Biol. Evol. 33, 885-897 (2016).

115. Costanzo, M. et al. The genetic landscape of a cell. Science (80-. ). 327, 425431 (2010).

116. Cooper, V. S., Vohr, S. H., Wrocklage, S. C. \& Hatcher, P. J. Why genes evolve faster on secondary chromosomes in bacteria. PLoS Comput. Biol. 6, e1000732 (2010).

117. Eberhard, W. G. Evolution in bacterial plasmids and levels of selection. Q. Rev. Biol. 65, 3-22 (1990).

118. Hug, L. A. et al. A new view of the tree of life. Nat. Microbiol. 1, 1-6 (2016).

119. Galata, V., Fehlmann, T., Backes, C. \& Keller, A. PLSDB: A resource of complete bacterial plasmids. Nucleic Acids Res. 47, D195-D202 (2019).

120. San Millan, A. \& MacLean, R. C. Fitness Costs of Plasmids: a Limit to Plasmid Transmission. Microbiol. Spectr. (2017) doi:10.1128/microbiolspec.MTBP0016-2017.

121. Vogwill, T. \& Maclean, R. C. The genetic basis of the fitness costs of antimicrobial resistance: A meta-analysis approach. Evol. Appl. 8, 284-295 (2015).

122. Salje, J. Plasmid segregation: How to survive as an extra piece of DNA. Critical Reviews in Biochemistry and Molecular Biology vol. 45 296-317 (2010).

123. Harrison, E. \& Brockhurst, M. A. Plasmid-mediated horizontal gene transfer is a coevolutionary process. Trends Microbiol. 20, 262-267 (2012).

124. Stewart, F. M. \& Levin, B. R. The population biology of bacterial plasmids: a priori conditions for the existence of conjugationally transmitted factors. Genetics 87, (1977).

125. San Millan, A., Heilbron, K. \& MacLean, R. C. Positive epistasis between coinfecting plasmids promotes plasmid survival in bacterial populations. ISME J. 8, 601-612 (2014).

126. Bouma, J. E. \& Lenski, R. E. Evolution of a bacteria/plasmid association. Nature 335, 351-352 (1988).

The first demonstration of compensatory evolution in a bacterialplasmid association. 
127. Harrison, E., Guymer, D., Spiers, A. J., Paterson, S. \& Brockhurst, M. A. Parallel Compensatory Evolution Stabilizes Plasmids across the ParasitismMutualism Continuum. Curr. Biol. 25, 2034-2039 (2015).

128. Wein, T., Hülter, N. F., Mizrahi, I. \& Dagan, T. Emergence of plasmid stability under non-selective conditions maintains antibiotic resistance. Nat. Commun. 10, 1-13 (2019).

129. San Millan, A. et al. Positive selection and compensatory adaptation interact to stabilize non-transmissible plasmids. Nat. Commun. 5, 5208 (2014).

130. Bottery, M. J., Wood, A. J. \& Brockhurst, M. A. Adaptive modulation of antibiotic resistance through intragenomic coevolution. Nat. Ecol. Evol. 1, 1364-1369 (2017).

131. Li, L. et al. Plasmids persist in a microbial community by providing fitness benefit to multiple phylotypes. ISME J. 14, 1170-1181 (2020).

132. Alonso-del Valle, A. et al. The distribution of plasmid fitness effects explains plasmid persistence in bacterial. bioRxiv https://www.biorxiv.org/content/10.1101/2020.08.01.230672v1 (2020) doi:10.1101/2020.08.01.230672.

133. Jordt, $\mathrm{H}$. et al. Coevolution of host-plasmid pairs facilitates the emergence of novel multidrug resistance. Nat. Ecol. Evol. (2020) doi:10.1038/s41559-0201170-1.

134. Hall, J. P. J., Wood, A. J., Harrison, E. \& Brockhurst, M. A. Source-sink plasmid transfer dynamics maintain gene mobility in soil bacterial communities. Proc. Natl. Acad. Sci. U. S. A. 113, 8260-8265 (2016).

135. Stalder, T. et al. Evolving Populations in Biofilms Contain More Persistent Plasmids. Mol. Biol. Evol. 37, 1563-1576 (2020).

136. Ridenhour, B. J. et al. Persistence of antibiotic resistance plasmids in bacterial biofilms. Evol. Appl. 10, 640-647 (2017).

137. Lopatkin, A. J. et al. Persistence and reversal of plasmid-mediated antibiotic resistance. Nat. Commun. 8, 1689 (2017).

138. Taddei, F. et al. Role of mutator alleles in adaptive evolution. Nature 387, 700-702 (1997).

139. San Millan, A. Evolution of Plasmid-Mediated Antibiotic Resistance in the Clinical Context. Trends in Microbiology vol. 26 978-985 (2018).

140. Arredondo-Alonso, S. et al. Plasmids shaped the recent emergence of the major nosocomial pathogen Enterococcus faecium. MBio 11, (2020). 
141. Ramsay, J. P. \& Firth, N. Diverse mobilization strategies facilitate transfer of non-conjugative mobile genetic elements. Current Opinion in Microbiology vol. 38 1-9 (2017).

142. Barry, K. E. et al. Don't overlook the little guy: An evaluation of the frequency of small plasmids co-conjugating with larger carbapenemase gene containing plasmids. Plasmid 103, 1-8 (2019).

143. Peña-Miller, R., Rodríguez-González, R., Maclean, R. C. \& San Millan, A. Evaluating the effect of horizontal transmission on the stability of plasmids under different selection regimes. Mob. Genet. Elements 5, 29-33 (2015).

144. Clark, A. J. \& Warren, G. J. Conjugal Transmission of Plasmids. Annu. Rev. Genet. 13, 99-125 (1979).

145. Chen, J. et al. Genome hypermobility by lateral transduction. Science (80-. ). 362, 207-212 (2018).

146. Halary, S., Leigh, J. W., Cheaib, B., Lopez, P. \& Bapteste, E. Network analyses structure genetic diversity in independent genetic worlds. Proc. Natl. Acad. Sci. U. S. A. 107, 127-132 (2010).

147. Erdmann, S., Tschitschko, B., Zhong, L., Raftery, M. J. \& Cavicchioli, R. A plasmid from an Antarctic haloarchaeon uses specialized membrane vesicles to disseminate and infect plasmid-free cells. Nat. Microbiol. 2, 1446-1455 (2017).

148. Abe, K., Nomura, N. \& Suzuki, S. Biofilms: Hot spots of horizontal gene transfer (HGT) in aquatic environments, with a focus on a new HGT mechanism. FEMS Microbiol. Ecol. 1-12 (2020) doi:10.1093/femsec/fiaa031.

149. Dubey, G. P. \& Ben-Yehuda, S. Intercellular nanotubes mediate bacterial communication. Cell 144, 590-600 (2011).

150. Porse, A., Schou, T. S., Munck, C., Ellabaan, M. M. H. \& Sommer, M. O. A. Biochemical mechanisms determine the functional compatibility of heterologous genes. Nat. Commun. 9, (2018).

151. Sánchez-Osuna, M., Cortés, P., Barbé, J. \& Erill, I. Origin of the mobile dihydro-pteroate synthase gene determining sulfonamide resistance in clinical isolates. Front. Microbiol. 10, (2019).

152. Guo, Q. et al. Glutathione-S-transferase FosA6 of Klebsiella pneumoniae origin conferring fosfomycin resistance in ESBL-producing Escherichia coli. J. Antimicrob. Chemother. 71, 2460-5 (2016).

153. Kirby, R. Evolutionary origin of the class $A$ and class $C \beta$-lactamases. J. Mol. Evol. 34, 345-350 (1992). 
154. Woodford, N. \& Ellington, M. J. The emergence of antibiotic resistance by mutation. Clinical Microbiology and Infection vol. 13 5-18 (2007).

\section{Acknowledgements}

This work was supported by the European Research Council (ERC) under the European Union's Horizon 2020 research and innovation programme (ERC grant agreement 757440-PLASREVOLUTION) and by the Instituto de Salud Carlos III (grant PI16-00860) cofunded by European Development Regional Fund "away to achieve Europe". A.S.M. is supported by a Miguel Servet Fellowship (MS15- 00012). J.R.-B. is a recipient of a Juan de la Cierva-Incorporación Fellowship (IJC2018035146-I) cofunded by Agencia Estatal de Investigación del Ministerio de Ciencia e Innovación. R.C.M. is supported by Wellcome Trust Grant (106918/Z/15/Z). A.S.M, J.R.-B. and R.L.-S. are members of the Spanish Consortium for Research on Epidemiology and Public Health (CIBERESP).

\section{Author contributions}

All authors contributed substantially to the conceptualization and writing of the manuscript, which was originally conceived and drafted by J.R.-B. and A.S.M.

\section{Competing interests}

The authors declare no competing interests.

\section{Peer review information}


Nature Reviews Microbiology thanks T. Dagan, E. Koonin, and E. Top, who coreviewed with S. Castaneda Barba and $\mathrm{C}$. Elg, for their contribution to the peer review of this work.

Figure 1. Plasmid prevalence and types. a| Phylogenetic tree highlighting the widespread distribution of known plasmids in the most relevant prokaryotic phyla. The outer track surrounding the tree is colour-coded according to the percentage of prokaryotic classes within each phylum that contain plasmids (see the plasmid prevalence key). The inner track delineates phyla depicted in the tree. Note that only four major prokaryotic phyla lack plasmids. The tree was built using 1,688 sequences obtained from a previously published concatenated ribosomal protein alignment ${ }^{118}$ and includes every prokaryotic phylum with at least 50 genomes deposited in the $\mathrm{NCBI}$ database. b| Plot showing the distribution of plasmid sizes for representative bacterial families and uncultured bacteria, showing the common bimodal (or multimodal) distribution of plasmid sizes. Red dashed line represents the local minor density point between the highest peaks for each bacterial family. Data was retrieved from the PLSDB plasmid database ${ }^{119}$.

Figure 2. Evolutionary forces that drive the evolution of plasmid-encoded genes. al Scheme summarising the evolutionary consequences of the multi-copy nature of plasmids. After the emergence of allele A (orange plasmid) in the plasmid pool of the top cell, plasmids are randomly segregated to daughter cells at each cell division. This simple process leads to the generation of heteroplasmid cells (green shading), carrying an allelic mixture. In some cells, mutation A is lost to segregational drift (blue shading). If a second independent mutation appears (mutation B; green plasmids) plasmid interference between mutations $A$ and can B occur. Eventually, mutation A reaches fixation, leading to homoplasmid mutant cells (red shading). b| Frequency of a plasmidic beneficial allele under positive selection over time in a bacterial population. A higher plasmid copy number increases the fixation time of the allele, but also the time in which both novel and ancestral alleles coexist under heteroplasmy (depicted as a dotted line). c| Only heteroplasmid cells carrying 
ancestral and evolved alleles can survive under periodic or simultaneous selection for both alleles.

Figure 3. The role of genetic dominance in plasmid evolution. al Dominance relationship among three alleles of the same gene. The allele $A$ (green) is dominant over alleles B (blue) and C (orange). Allele B is dominant over allele C. b| The phenotypic effect of new alleles will depend on their degree of genetic dominance. If a new plasmid allele emerges by mutation (left) or is acquired through horizontal gene transfer (HGT; right) it will only produce a phenotype if it is dominant over the resident alleles. $\mathbf{c |}$ Fitness effect of mutations depending on the number of plasmids carrying the mutant allele for dominant mutations (blue line), and recessive mutations (red line). d| Effect of genetic dominance on phenotypic mutation rates for plasmidencoded genes. The plot shows the mutation rates for a plasmid-carried gene at different copy numbers compared with a chromosomal copy of the same gene (blue line). The frequency of mutants able to produce a phenotype increases with plasmid copy number for mutations of high dominance, but decreases for mutations of low dominance. Part $d$ is adapted with permission from REF. ${ }^{102}$.

\section{Box 1. The plasmid paradox}

Plasmid carriage imposes a burden on bacterial physiology, which often translates into a reduction in bacterial fitness ${ }^{52,120,121}$. Plasmid costs can originate both as a consequence of plasmid life cycle (for example, plasmid conjugation, replication or gene expression), as well as from the interactions of the plasmid or plasmid-encoded proteins with the host bacterium ${ }^{120}$. Fitness costs associated with plasmids are arguably the main limit to plasmid persistence in bacterial populations. Despite a number of effective mechanisms designed to ensure a correct plasmid segregation (such as active partitioning systems ${ }^{122}$ and post-segregational killing systems ${ }^{5}$ ), the probability of plasmid loss during cell division is higher than zero. In the absence of selection for plasmid-encoded traits, the plasmid-free cells that emerge due to imperfect plasmid segregation will eventually outcompete plasmid-carrying bacteria, 
and only those plasmids with a particularly high conjugation rate can persist as genetic parasites. In the presence of constant selection for plasmid-encoded traits, beneficial genes may eventually integrate into the bacterial chromosome, rendering the plasmid dispensable 26,104 . Consequently, it is difficult to explain the widespread prevalence of plasmids in bacterial populations, a puzzle known as the plasmid paradox'123.

Since the pioneering work of Stewart and Levin ${ }^{124}$, multiple studies have explored the population biology principles that explain the stable maintenance of plasmids in bacterial populations. Some key discoveries from those studies are helping to solve the plasmid paradox. First, the costs of plasmids can be alleviated over time through mutations in either plasmids or chromosomes (compensatory evolution [G]), or through the acquisition of further plasmids ${ }^{125}$. Compensatory evolution leads to the formation of stable bacteria-plasmid associations ${ }^{126-129}$, and in some cases can lead to co-dependence ${ }^{130}$. Second, the presence of 'cost-free' hosts promotes the maintenance of plasmids in complex communities ${ }^{131-133}$, for example by recurrent transfer from cost-free hosts to species where plasmids are unstable ${ }^{134}$. Third, certain bacterial lifestyles, such as growth within biofilms, can promote plasmid persistence ${ }^{135,136}$. Fourth, many plasmids may indeed conjugate at high enough rates to be maintained in the absence of selection ${ }^{137}$. Fifth, the multi-copy nature of plasmids allows the formation of transient plasmid forms, such as satellite plasmids ${ }^{87}$, which reduce the costly expression of accessory genes. Finally, some of the plasmidmediated evolution mechanisms described in this Review may contribute to plasmid persistence in bacterial populations through second order selection [G], when plasmids are maintained by virtue of their association to high fitness bacterial clones that they generate, for example when mutations caused by plasmid-carried DNA polymerases generate beneficial mutations in the bacterial chromosome ${ }^{138}$. 


\section{Box 2. Mechanisms for plasmid mobility}

There are several mechanisms for plasmid mobility (see the figure):

- Conjugation is the principal mechanism of plasmid mobility and requires cellto-cell contact involving pilus formation ${ }^{9}$. Conjugation can occur between phylogenetically distant organisms and several studies highlight this mechanism as a key factor in the spread of antibiotic resistance $19,29,139,140$.

- Conjugative mobilization allows mobilizable plasmids, which lack part of the conjugation machinery, to be transferred by the conjugative apparatus of other conjugative elements in the cell. Recent reports suggest plasmid mobilization as an important mechanism of plasmid spread ${ }^{6,141-143}$. Non mobilizable plasmids can be additionally transferred through plasmid conduction $^{144}$. Conduction occurs through the co-integration via recombination of non-mobilizable and mobilizable or conjugative plasmids. The co-integrate plasmid is then transferred to a new recipient where it can be resolved.

- Plasmid transduction occurs when a bacteriophage (phage) packages plasmid DNA in viral particles and infects another bacterium. Once inside the new host, the plasmid can excise and re-circularize again. Phages are the most abundant gene-transfer particles, and new findings strongly suggest that they are likely to have an important role in horizontal gene transfer (HGT) between closely related bacteria ${ }^{145}$, but the extent to which transduction contributes to long distance HGT remains unclear ${ }^{146}$. 
- Plasmid transformation is the uptake of free plasmid DNA by organisms. This mechanism is expected to occur in low rates constrained by the presence of free plasmids in the environment and by the physiology of the host ${ }^{9}$.

- Recent reports show the presence of other non-canonical mechanisms of plasmid spread. Some plasmids use specialized vesicles to disseminate in a process known as vesiduction ${ }^{147}$, which is hypothesized to occur at high rates in biofilms ${ }^{148}$. Plasmid transmission via intracellular connections mediated by nanotubes has also been suggested ${ }^{149}$.

\section{Box 3. Genetic dominance shapes plasmid-mediated antibiotic resistance}

Upon the massive introduction of antibiotics in clinical practice, some chromosomal resistance genes have been mobilised to plasmids, where they uncontrollably spread among pathogenic bacteria. But, why do some antibiotic resistance genes remain associated to chromosomes whereas others are successfully transferred? Among the multiple factors that may limit transferability of antibiotic resistance genes ${ }^{150}$, genetic dominance stands out as one of the major forces explaining the commonly observed divergence between chromosomal and plasmid resistance alleles ${ }^{102}$.

Several examples highlight that dominant versions of housekeeping antibiotic resistance genes (that is, those able to confer resistance regardless of the genetic context) have been successfully transferred from chromosomes to plasmids. Perhaps the clearest case is sulfonamide resistance sul1 and sul2 genes, which originated from a mobilization event of housekeeping folP genes present in Rhodobiaceae and Leptospiraceae families ${ }^{151}$. Other examples include the fosfomycin resistance fos $A$ gene, which originated from a chromosomal glutathione-S-transferase of Klebsiella 
pneumoniae ${ }^{152}$, or class $A \beta$-lactamases that likely derived from penicilling-binding proteins of actinomycetes ${ }^{153}$.

On the contrary, other resistance determinants are completely absent from bacterial plasmids, such as the antibiotic resistance alleles of $r p s L, r p o B$ or gyrA, which confer resistance to streptomycin, rifampicin and quinolones, respectively ${ }^{154}$. These alleles are recessive to the wild-type sensitive variant, which is naturally present in most bacterial chromosomes. Consequently, if these alleles were transferred by plasmids, they would not confer any resistance to the recipient bacteria ${ }^{94,101,102}$, a fact that likely explains why they are exclusively found on chromosomes.

Obtaining knowledge of the degree of dominance of a given resistance allele is thus crucial to predict which antibiotic resistance genes are likely to spread horizontally and to rationally assess the risk of spread for resistance alleles to novel antibiotics.

\section{Box 4. Outstanding questions}

- Why are plasmids extremely prevalent in most bacterial taxa but absent from others? Why are microbial eukaryotes relatively depleted of plasmids?

- Do the evolutionary benefits associated with plasmids offset the costs imposed by their parasitic lifestyles?

- How are antibiotic resistance genes mobilised to plasmids? Once a resistance gene becomes mobile, what are the forces that shape its evolution?

- Can we selectively eliminate resistance plasmids from complex bacterial communities?

- The function of most plasmid genes is not known ${ }^{11}$. Does this plasmid 'dark matter' contain genes that are beneficial, or is it simply parasitic DNA that spreads by virtue of association with successful plasmid replicons? How does plasmid ‘dark matter' alter evolvability? 


\section{Glossary}

Horizontal gene transfer: Transfer of genetic material between cells that do not share an ancestor-descendant relationship.

Post-segregational killing systems: Genetic systems that ensure plasmid maintenance. They typically rely on the production of a long-lasting toxin and a shortlived antitoxin. If the plasmid is lost in a daughter cell, the antitoxin is rapidly degraded and the stable toxin kills the plasmid-free cell.

Type IV CRISPR-Cas systems: A recently characterized type of CRISPR-Cas system that are found predominantly on plasmids and primarily target other plasmids. Type IV CRISPR-Cas systems are thus believed to have a role in mediating interplasmid competition.

Plasmid segregation: Physical separation of plasmid molecules to be inherited by daughter cells during cell division.

Tandem genetic duplications: Duplication of a region of DNA adjacent to the original one.

Bet-hedging: Stochastic process by which some individuals in a community are better suited to tackle environmental perturbations, usually at the price of a reduced growth rate in the short term.

Recombination: Exchange of genetic information between two distinct DNA molecules.

Mutator strains: Strains that permanently show unusually high mutation rates due to a malfunction of a DNA repair mechanism. 
Transposable elements: DNA sequences that can move within genomes by a cutand-paste mechanism.

Heteroplasmy: Co-existence of two different plasmids sharing the same nucleotide sequences for all regions involved in replication and maintenance system within the same cell. Cells carrying plasmids under heteroplasmy are dubbed heteroplasmid cells, whereas cells carrying a unique version of a plasmid are termed homoplasmid cells.

Genetic drift: Change in allele frequency in a population due to random sampling.

Clonal interference: Competition between cellular lineages in a population arising from different beneficial mutations in asexually reproducing organisms.

Standing genetic variation: The presence of more than one allele at a locus in a population before environmental change.

Trade-off: In evolution, trade-offs are negative correlations between ancestral and novel traits.

Evolutionary innovation: Evolutionary changes that allow a species to expand its ecological niche and thrive in new environments.

SOS stress response: Coordinated cellular response to genotoxic stress which involves the expression of more than 40 genes whose main function is to repair damaged DNA.

Gene dosage effects: Effect by which the phenotype of a given mutation is proportional to the cumulative number of mutant alleles present in the cell.

Integron: Genetic element composed by an integrase gene and a recombination site in which gene cassettes can be directionally integrated or excised by integrasemediated site-specific recombination. 
Purifying selection: Selective pressure that eliminates deleterious alleles from populations.

Compensatory evolution: Process by which the fitness cost produced by the acquisition of a plasmid is ameliorated through mutations in the chromosome and/or the plasmid.

Epistatic interactions: Phenomenon by which the phenotypic contribution of a gene varies depending on the presence or absence of another gene. The phenotypic effect of both genes in combination is thus different than the expected according to the phenotypes they conferred separately.

Second order selection. Process by which evolution, while directly selecting for adaptive genetic variability, indirectly selects for the system that created that variability.

\section{Table of contents blurb}

Recent studies have revealed that the evolutionary impact of plasmids goes above and beyond being mere gene delivery platforms. In this Review, Rodríguez-Beltrán, San Millán and colleagues discuss the advances that underscore the importance of plasmids in bacterial ecology and evolution beyond horizontal gene transfer. 